\title{
Maturational and gender differences in rodent escape strategies: Possible implications for preclinical models of anxiety
}

\author{
SHEILA M. KING \\ University College London, London, England
}

\begin{abstract}
This study profiles two clearly dissociable escape strategies (jumping and backing off) in a newly established preclinical model of extreme anxiety (i.e., the unstable elevated plus maze) and examines the contribution of ontogeny and gender to the behavioral profiles exhibited. Clear age-related differences in escape behavior were found: Although the younger animals exhibited all the preparatory behaviors appropriate to flight from the apparatus-that is, attempting to back off, backtracking, preparing to jump, turning, and scanning - the older animals more readily translated these into actual escape. There were no significant sex-related differences in escape rates or in three of the escape-related behaviorsthat is, backtracking, attempting to back off, and preparing to jump. Clear dissimilarities were, however, found in a number of other escape-linked behaviors: Females scanned, end-reached, and turned more than males. These results indicate that ontogeny and gender significantly contribute to the behaviors exhibited in aversive situations and might explain at least some of the inconsistent results frequently obtained in preclinical models of anxiety.
\end{abstract}

Early behavioral studies have demonstrated that agerelated changes in fear responses to aversive stimuli follow a similar pattern in various species (monkeys: Bernstein \& Mason 1962; Green, 1965; dogs: Melzack, 1954; birds: Kuhlmann, 1909; Schaller \& Emlen, 1962; rats: Bronstein \& Hirsch, 1976; Hard, Engel, \& Musi, 1982). Infant reactions are predominantly immobility and vocalization; at older ages, withdrawal/retreat behavior appears, and still older animals show flight and escape responses. Similarly, sex differences in avoidant/defensive responding have been shown in diverse animals (monkeys: Crepeau \& Newman, 1991; rats: D. C. Blanchard \& R. J. Blanchard, 1990; D. C. Blanchard, R. J. Blanchard, Tom, \& Rodgers, 1990; D. C. Blanchard, Shepherd, Carobrez, \& R. J. Blanchard,199I; R. J. Blanchard \& D. C. Blanchard, 1989; R. J. Blanchard, D. C. Blanchard, \& Weiss, 1990; R. J. Blanchard, Yudko, Rodgers, \& D. C. Blanchard, 1993; Scouten, Grotelueschen, \& Beatty, 1975; frogs: Murphey, Becak, \& Dias de Moraes, 1981). In a review of the early literature, Archer (1975) suggested that rats show different sex-typical fear responses, females being more prone to active escaping and males to immobility. More recently, the Blanchards and their co-workers have carried out a number of studies that show that female rats exhibit consistently higher levels of defensive behavior than do males in situations involving potential threat (D. C. Blanchard \& R. J. Blanchard, 1990; D. C. Blanchard et al., 1990; D. C.

This study was supported by the Royal Society Robert and Joan Case Research Fellowship. Correspondence concerning this article should be addressed to S.M. King, Department of Psychology, University College London, Gower Street, London WCIE 6BT, England (e-mail: s.king@ucl.ac.uk).
Blanchard et al., 1990; D. C. Blanchard et al., 1991; R. J. Blanchard \& D. C. Blanchard, 1989; R. J. Blanchard et al., 1990; R. J. Blanchard et al., 1993), although not to actual approach/contact by a discrete threat stimulus (D. C. Blanchard \& R. J. Blanchard, 1990; R. J. Blanchard, Flannelly, \& D. C. Blanchard, 1986).

Apart from the work carried out by the Blanchards and their coworkers, however, developmental and gender differences have been relatively neglected by neurobiological research into defensive behaviors in recent years. Given the widespread use in the field of psychopharmacology of behavioral tests designed to measure levels of anxiety/fear in rodents in response to aversive stimulation and the equally widespread and inconsistent results obtained from many of these studies, it seemed timely to provide a systematic analysis of age- and sex-related changes in rats in response to exposure to a highly aversive situation. This was achieved by studying the ontogenetic and gender effects on the repertoire of escape-related behaviors exhibited in a newly established preclinical model of extreme anxiety - that is, the unstable elevated exposed plus maze (UEEPM; King, 1999a, 1999b).

This model capitalizes on the innate aversion of many species to instability, height, and exposure, and accumulating evidence from behavioral and electrical stimulation studies (King, 1999a, 1999b) suggests that the flight behaviors exhibited on the UEEPM are analogous to the chronically hyperaroused, highly avoidant, flight-oriented state exhibited by patients suffering from one of the more extreme fear-related clinical disorders. A clear positive correlation has been reported between the propensity to escape from the UEEPM and well-validated anxiety measures on the standard elevated plus maze. Principal com- 
Table 1

\begin{tabular}{lcccccccccc}
\multicolumn{1}{c}{ Mean Weight (in Grams) of Each Age Group by Sex } \\
\multicolumn{1}{c}{} & $5-10$ & Weeks & \multicolumn{1}{c}{$15-20$ Weeks } & $25-30$ Weeks \\
Sex & $M$ & $S E$ & $n$ & $M$ & $S E$ & $n$ & $M$ & $S E$ & $n$ \\
Female & 194 & 13 & 22 & 264 & 6 & 20 & 268 & 6 & 22 \\
Male & 290 & 21 & 22 & 483 & 9 & 19 & 499 & 8 & 22
\end{tabular}

ponents analyses have identified separate and independent factors relating to these two sets of behaviors, which suggests that the escape-linked behaviors exhibited on the UEEPM reflect a different type of anxiety from that exhibited on the standard elevated plus maze. It has been demonstrated, moreover, that direct activation of the rodent midbrain defense system by repetitive electrical stimulation significantly increases the levels of these escaperelated behaviors over a 3-month period, a long-term alteration in behavior similar to the persistent fight-orflight mode observed in many pathological anxiety states.

Pharmacological validation of the model has also recently been reported (King, Green, \& Vale, 1999). The 5-HT receptor agonist meta-chlorophenylpiperazine (mCPP), an anxiogenic agent used to induce panic attacks for diagnostic purposes, was found to increase the propensity to escape from the UEEPM (i.e., it had a putative anxiogenic effect), whereas the benzodiazepine receptor agonist chlordiazepoxide (CDP), an established anxiolytic agent that is widely prescribed for anxiety, decreased this propensity (i.e., it had a putative anxiolytic effect).

\section{METHOD}

\section{Subjects}

One hundred twenty-seven Hooded Lister rats (64 female, 63 male), bred in the laboratory, were used as subjects. These were divided on the basis of age into three groups: 5-10 weeks, 15-20 weeks, and 25-30 weeks (see Table 1 for mean weights). They were housed in same-sex pairs in clear polycarbonate cages, with food (standard rat pellets) and water freely available. The rat-keeping room was maintained at a controlled humidity $(62 \% \pm 5 \%)$ and temperature $\left(23^{\circ} \pm 1^{\circ} \mathrm{C}\right)$ and was run on a 12 -h light:dark cycle.

\section{Apparatus and Procedure}

Testing was conducted in a room separate from the keeping room. Ambient lighting was provided by a diffuse ceiling light strip, the illuminance being similar to that in the keeping room during the light cycle. The apparatus was completely surrounded by a tall $(100 \mathrm{~cm}$ high) matt black cloth screen to provide a uniform surround and to minimize the possibility of distraction created by visual cues. The rats were tested during their 12-h light cycle. Immediately prior to testing, the rats were brought to the experimental room in their home cages. Individual rats were placed in the middle of the testing apparatus, and their behavior was recorded throughout the trial by a video camera mounted directly above the apparatus, linked to a monitor and a VCR. At the conclusion of the testing period, the rat was returned to the home cage, and the apparatus was cleaned with a solution of $20 \%$ ethanol and was dried before the next animal was tested. The animals were not handled, other than to transfer them from the home cage to the test environment.

Unstable elevated plus maze. The apparatus used was a black, perspex plus-shaped maze, elevated $50 \mathrm{~cm}$ above the floor. Each arm measured $50 \times 10 \mathrm{~cm}$, and the junction of the four arms mea- sured $10 \times 10 \mathrm{~cm}$. All four arms of the maze were exposed, with only a small perspex ledge $(0.5 \mathrm{~cm}$ high $)$ surrounding each arm for the animals to grip. The floor of the plus maze was covered with matt black rubber to reduce slippage. A motor oscillated the elevated platform in the horizontal plane at a rate of $85 \mathrm{revs} / \mathrm{min}$, with a movement amplitude of $2 \mathrm{~cm}$ each side of the central point. The rat was placed in the center of the maze, the motor was started after an interval of approximately $3 \mathrm{sec}$, and the animal's behavior was monitored. If the rat lost its footing, the oscillating movement was stopped until all four feet were back on the platform. If the animal fell off, it was placed back on the maze and left for a few seconds on the stable maze before testing was resumed. The trial was terminated either when the animal escaped the apparatus or when a predefined time limit of 5 min was reached.

Behavioral measures. The majority of the behaviors measured have been described in detail in a previous paper (King, 1999a). In brief, these comprised the following.

\section{Escape-related behaviors}

1. Jumping off the apparatus: Jumping was easily differentiated from falling, since a jump was invariably preceded by preparing to jump (see [2] below), whereas a fall was usually preceded by foot slippage and scrabbling to stay on the apparatus.

2. Preparing to jump: A foreshortening of the body, with the back legs drawn up under the hindquarters, and leaning the forequarters over the side of the apparatus.

3. Scanning: Protruding the head over the exposed sides of the arms and scrutinizing in any direction.

4. End-reaching: The number of times the animal reached the end of any arm.

5. Rotating in $90^{\circ}$ movements, either in the central area or on one of the open arms.

A further group of behaviors was noted from some animals that achieved the same goal as jumping-- that is, escaping the apparatusbut were clearly dissociable from jumping and, hence, were categorized separately.

6. Backing off the apparatus: During testing some animals shifted off the apparatus hindquarters first, often turning their heads and peering over their forequarters while so doing. Before dropping from the apparatus, some animals would hang from the edge of the arm with their forepaws, possibly to foreshorten their fall.

7. Attempts to back off: A behavior similar to (6) above, except that the action of backing off was reversed just before the animal's center of gravity was shifted irreversibly over the arm; often accompanied by a scrabbling of the forepaws.

8. Backtracking: A foreshortening of the body, quickly followed by stepping backward along the maze arms. This action frequently culminated in a $90^{\circ}$ turn, positioning the animal at right angles to the maze arm, followed by the animal's either backing off or attempting to back off the apparatus.

\section{Exploratory/motor behaviors}

1. Crossing zones: crossing from one of the nine zones (defined as the central area, the inner portion of the arms $\times 4$, and the outer portion/end of the arms $\times 4$ ) to another with all four paws. Note that this division of the apparatus differs from that in previous experiments, in which the apparatus was divided into only five zones (defined as the four arms and the central area; King, 1999a, 1999b).

2. Time spent in the center of the maze, expressed as a ratio of the total time spent on the apparatus.

It was noted during testing that some animals spent a large proportion of the test time at the ends of the arms, frequently with their hindquarters protruding over the edge of the apparatus. One further category was, therefore, added to the existing range of behaviors.

3. Time spent at the end (defined as the outermost third) of any of the maze arms, expressed as a ratio of the total time spent on the apparatus. 

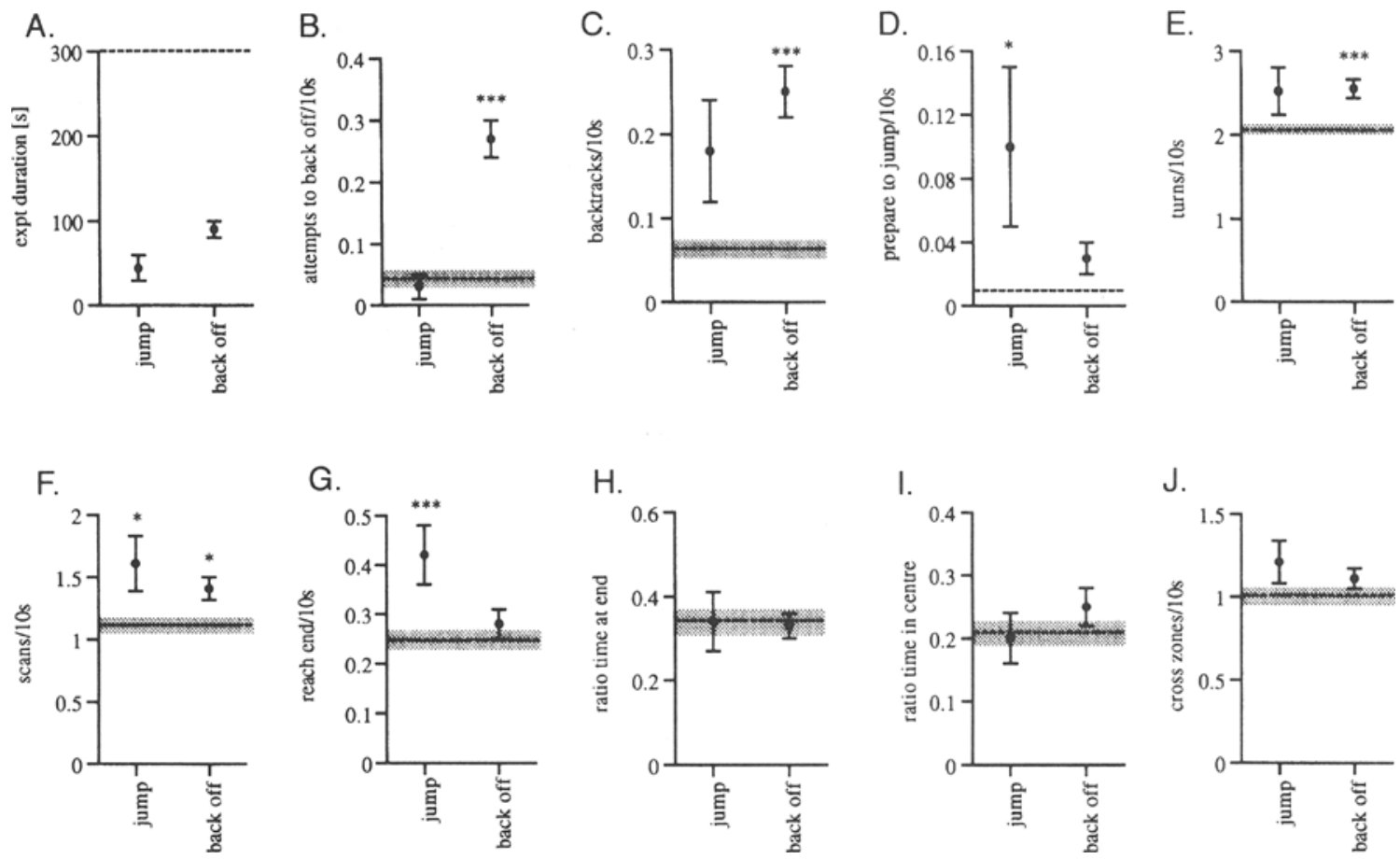

Figure 1. Means $( \pm S E)$ of escape-related and motor/exploratory behaviors for the animals that jumped off $(n=16)$ and those that backed off $(n=51)$ the unstable elevated plus maze. The horizontal dashed line indicates the group mean ( $\pm S E$ : gray shaded area) for those animals that remained on the apparatus throughout the trial-that is, the no-escape group ( $n=$ 60). *Significantly different from the no-escape group $(p<.05$; Mann-Whitney $U$ test $) . * *$ Significantly different from the no-escape group ( $p<.001 ;$ Mann-Whitney $U$ test).

\begin{abstract}
Statistical Analysis
To determine whether the behavioral profiles of the animals that jumped or backed off the unstable plus maze differed from those of the animals that remained on the apparatus throughout the trial, all the dependent measures were assessed with the Mann-Whitney $U$ test. To assess whether there were significant age- and sex-related differences in the propensity to escape from the apparatus, frequencies of jumping and backing off were analyzed with the chi-square test of association; all other dependent measures were assessed with Kruskal-Wallis one-way analysis of variance. An alpha level of .05 was used for all the statistical tests.
\end{abstract}

\section{RESULTS}

This experiment was terminated either when the animal escaped (jumped or backed off) the unstable maze or after $5 \mathrm{~min}$ had elapsed; hence, the duration of the experiment varied between animals. To aid in comparison of the results, therefore, the data for all the behaviors exhibited on the apparatus (other than those for escaping and experiment duration) were converted into average frequencies over $10 \mathrm{sec}$. Furthermore, to guard against misleading comparisons being drawn between the frequencies of the behaviors exhibited by animals that escaped at an early stage of the experiment and those exhibited by animals that remained on the apparatus for the duration of the experiment, only the data from the first $120 \mathrm{sec}$ of the experiment, for all behaviors other than jumping/backing off and experiment duration, were included in the analyses. This time period is the same as that used in previous experiments (King, 1999a, 1999b). Finally, 5 animals (4 females, 1 male) jumped off the apparatus within $15 \mathrm{sec}$ of its starting to oscillate. Consequently, since there were no data from these animals for any of the remaining escape-related and locomotor behavior categories, they were excluded from these further analyses.

\section{Jumping Versus Backing Off: Differences in Behavioral Profiles}

Over all age groups, $12.6 \%(16 / 127)$ of the animals jumped off the apparatus before the end of the trial, as compared with $40.2 \%(51 / 127)$ that backed off. A comparison of the average experiment duration for these two groups reveals that the animals that jumped did so significantly sooner than did those animals that backed off $(U=174.0, p<.001$; see Figure 1A). To establish the behavioral profiles of the two escape groups - that is, those that jumped and those that backed off-the means for each group were compared separately with the group means of those animals that remained on the apparatus throughout the trial (the no-escape group; see Figures $1 \mathrm{~B}-1 \mathrm{~J})$.

Jump group versus no-escape group. An analysis of the group means revealed that the jumpers prepared to jump $(U=234.5, p<.05)$, scanned over the sides of the 

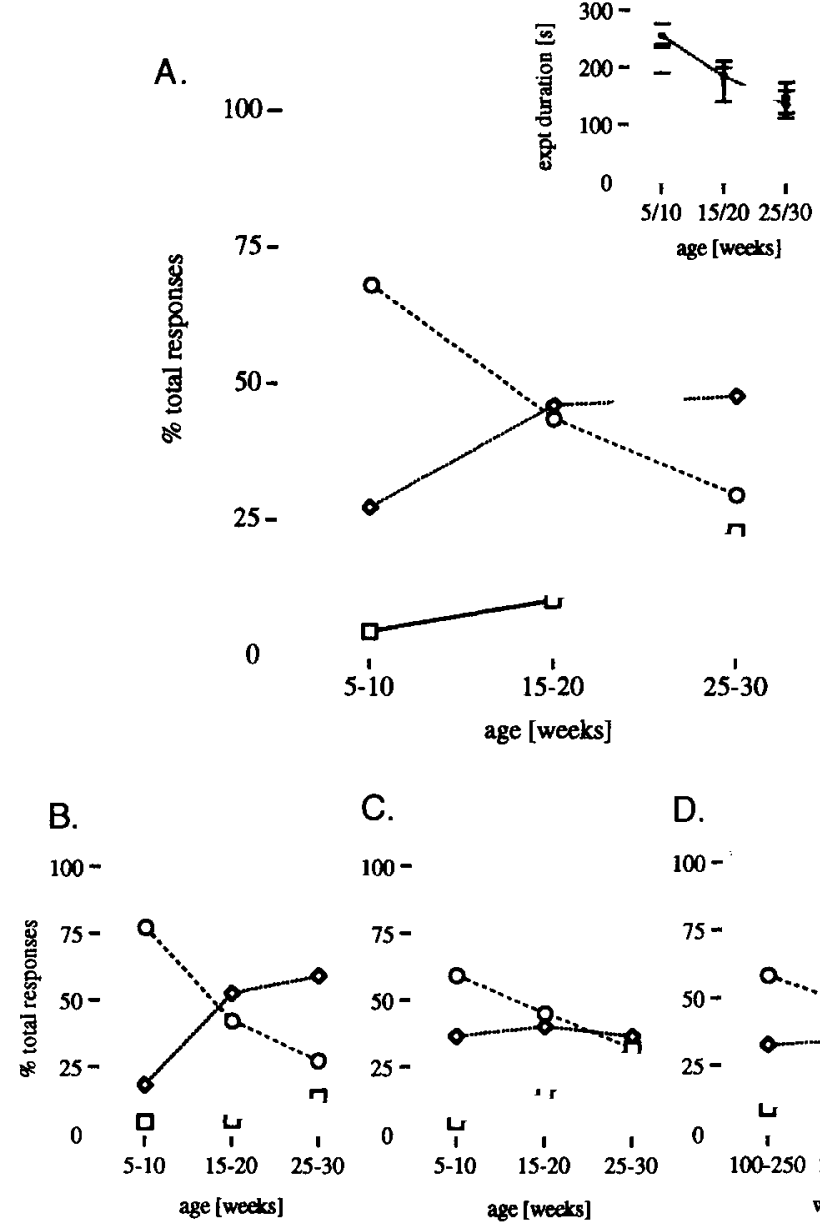

C.

D.

Figure 2. Panel A: pattern of escape behaviors as a percentage of total responses (square $=$ jump; diamond $=$ back off; circle $=$ no escape) for different age groups (both sexes combined). Means ( $\pm S E$ ) for experiment duration for different age groups and different sexes (females, solid line; males, dashed line; see inset, Figure 2A). Pattern of escape behaviors as a percentage of total responses for males only (panel B), females only (panel C), and different weight groups (panel D).

arms $(U=196.0, p<.05)$, and reached the end of the arms $(U=131.5, p=.001)$ significantly more frequently than did those that did not escape. There were no significant group differences in attempting to back off $(U=268.5$, $p=.25)$, backtracking $(U=248.0, p=.16), 90^{\circ}$ turns $(U=$ $218.5, p=.07)$, ratio of time spent in the center of the maze $(U=328.0, p=.97)$, ratio of time spent at the ends of the arms $(U=317.5, p=.84)$, or zone crossing $(U=$ $244.0, p=.17)$.

Back-off group versus no-escape group. An analysis of the group means revealed that those animals that backed off the apparatus also attempted to back off $(U=434.0$, $p<.001)$, backtracked $(U=624.0, p<.001)$, scanned over the sides of the arms $(U=1,156.0, p<.05)$, and made $90^{\circ}$ turns $(U=846.5, p<.001)$ significantly more frequently than did those that did not escape. There were no significant group differences in preparing to jump ( $U=$
$1,481.0, p=.61)$, reaching the end of the arms $(U=1,471.0$, $p=.72)$, crossing from one zone to another $(U=1,352.5$, $p=.29)$, ratio of time spent in the center of the maze ( $U=$ $1,403.0, p=.45)$, or ratio of time spent at the ends of the $\operatorname{arms}(U=1,434.0, p=.57)$.

\section{Patterns of Escape: \\ Maturation and Gender Effects}

Comparison of the rates at which the different age groups escaped the apparatus (see Figure $2 \mathrm{~A}$ ) reveals significant differences in escape behaviors $[\chi(2)=13.48$, $p=.001]$ : The propensity to escape increased very significantly with increasing maturity.

Commensurate with this finding, the average experiment duration decreased significantly with age $[\chi(2)=$ $13.29, p=.001$; see inset, Figure 2A]. Of those animals that escaped, the proportion of the animals that jumped off 
A.

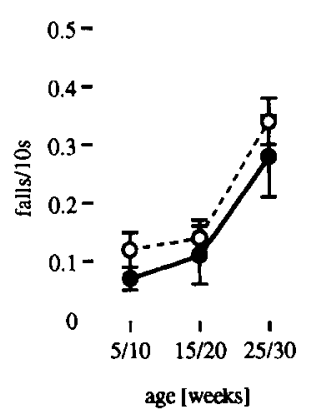

$\mathrm{F}$.

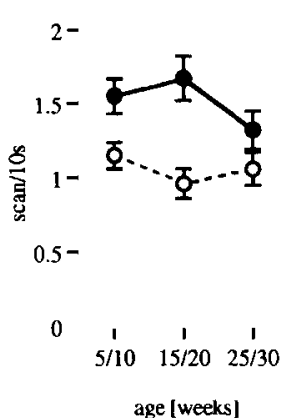

B.

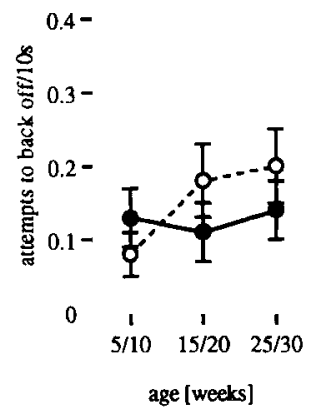

G.

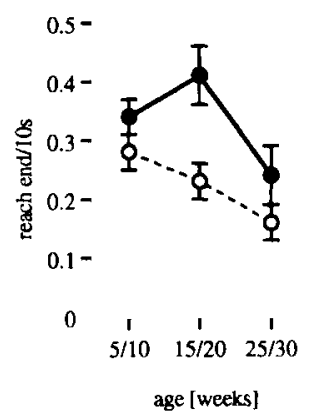

C.

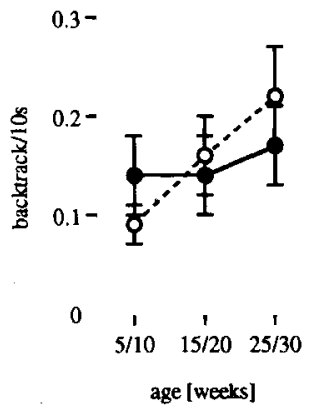

$\mathrm{H}$.

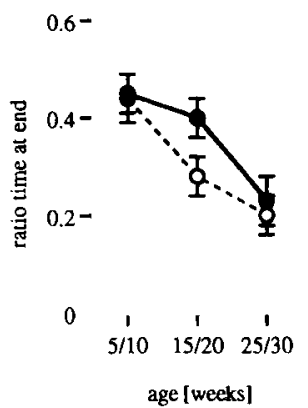

D.

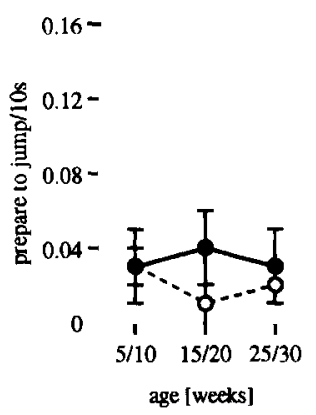

I.

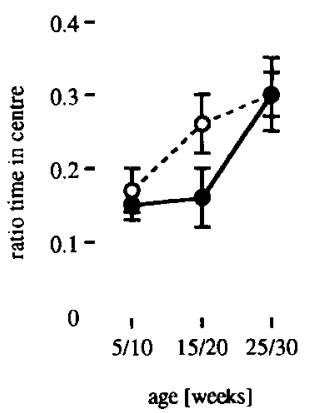

E.

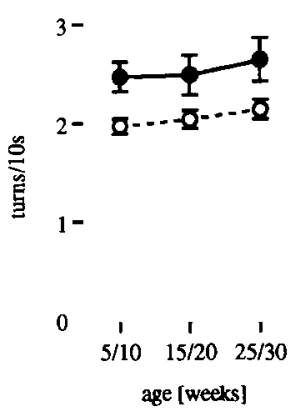

J. $1.5-$

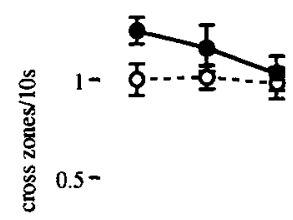

\begin{tabular}{cccc}
0 & 1 & 1 & 1 \\
$5 / 10$ & $15 / 20$ & $25 / 30$ \\
\multicolumn{3}{c}{ age [weeks] }
\end{tabular}

Figure 3. Means ( $\pm S E$ ) of escape-related and motor/exploratory behaviors for different age groups and different sexes (females, solid line, filled circle; males, dashed line, unfilled circle) on the unstable elevated plus maze.

increased significantly with age $[\chi(2)=6.88, p<.05]$, whereas the proportion of the animals that backed off did $\operatorname{not}[\chi(2)=4.67, p=.1]$. No significant overall differences were found in the rates at which males and females escaped the apparatus $[\chi(1)=0.19, p=.66$; see Figures $2 \mathrm{~B}$ and $2 \mathrm{C}]$ or in the rate at which they either jumped $[\chi(1)=$ $2.47, p=.12]$ or backed off the apparatus $[\chi(1)=0.38$, $p=.54]$. In line with these findings, no significant sex differences were found in experiment duration $[\chi(1)=1.06$, $p=.3$; see inset, Figure 2A]. Finally, in order to ascertain whether the increased propensity to escape with age could simply be explained in terms of weight differences, the data for all ages and both sexes were divided into three equal-sized groups on the basis of weight (100-250, $251-400$, and $401+g$ ), and their propensity to escape was compared (see Figure 2D). No significant weight-related differences were, however, found in escape rates $[\chi(2)=$ $4.67, p=.1]$.

Age-related differences in behavioral profiles. An analysis of the group means for the behaviors exhibited during the initial $120 \mathrm{sec}$ from the start of the experiment (see Figures 3A-3J) reveals that, with age, there was a significant decrease in the rate at which the animals reached the end of the arms $[\chi(2)=17.31, p<.001]$, a decrease in the ratio of time spent at the ends of the arms $[\chi(2)=$ $24.87, p<.001]$, and a corresponding increase in the ratio of time spent in the center of the maze $[\chi(2)=15.09, p<$ $.001]$. Falling off the apparatus also increased signifi- cantly with age $[\chi(2)=21.65, p<.001]$. In contrast, there were no significant age-related differences in preparing to jump $[\chi(2)=0.50, p=.77]$, attempting to back off $[\chi(2)=2.91, p=.23]$, backtracking $[\chi(2)=4.43, p=.1]$, scanning over the sides of the arms $[\chi(2)=3.71, p=.16]$, turning $[\chi(2)=0.62, p=.73]$, or zone crossing $[\chi(2)=$ $2.56, p=.28]$.

Sex-related differences in behavioral profiles. An analysis of the group means (see Figures 3A-3J) also reveals a number of significant sex-related differences in behavior: Females scanned over the sides of the arms $[\chi(1)=$ $20.7, p<.001]$, reached the end of the arms $[\chi(1)=9.47$, $p<.01]$, crossed zones $[\chi(1)=6.35, p=.01]$, and made $90^{\circ}$ turns $[\chi(1)=12.74, p<.001]$ significantly more frequently than did males. There were, however, no significant sex differences in preparing to jump $[\chi(1)=0.36$, $p=.55]$, attempting to back off $[\chi(1)=0.32, p=.57]$, backtracking $[\chi(1)=0.01, p=.98]$, ratio of time spent in the center of the maze $[\chi(1)=3.18, p=.07]$, or ratio of time spent at the ends of the arms $[\chi(1)=2.56, p=.11]$.

\section{DISCUSSION}

The aim of this study was to provide a systematic analysis of age- and sex-related changes in rats in response to exposure to a highly aversive situation. This was achieved by studying the ontogenetic and gender effects on the repertoire of escape-related behaviors in a newly 
developed preclinical model of extreme anxiety - that is, the UEEPM (King, 1999a, 1999b).

\section{Maturational Effects on Patterns of Escape}

Clear age-related differences in escape behavior were found: The older animals displayed a much greater propensity to escape than did the immature ones. This finding was largely accounted for by a greater proportion of the older animals jumping from the apparatus, as the percentage of the animals backing off the apparatus remained relatively stable. This incremental tendency to jump cannot simply be attributed to such factors as weight gain or an overall increase in motor activity, since no significant differences in rates of escape were found for different weight categories and the younger rats were at least as motorically active as the older animals. Indeed, apart from actual escape, the only consistently significant difference between younger and older animals' behavioral profiles was that the immature animals reached the ends of the apparatus more frequently than did the more mature animals, spent a greater proportion of their time there, and spent less time in the center of the maze. Insufficient motor skills can also be discounted as an explanation, inasmuch as Bolles and Woods (1964) have reported that, by 3 weeks of age, infant rats engage in various types of locomotor activity - for example, walking, running, jumping, and climbing, as well as fighting, playing, burrowing, and social grooming - which suggests that motor development in rats occurs well in advance of the changing patterns of fear reactions seen in this study. It appears, therefore, that although the younger animals may exhibit all the preparatory behaviors appropriate to flight from the apparatus - that is, attempting to back off, backtracking, preparing to jump, turning, and scanningthe older animals more readily translate these into actual escape.

The discovery of ongoing maturational changes in escape behaviors in peri- and post-pubertal animals may go some way toward explaining the discrepant results in many psychopharmacological studies of "fear"-motivated behaviors-for example, the widely used elevated plus maze (Handley \& Mithani, 1984). Strain differences (Rex, Sondern, Voigt, Franck, \& Fink, 1996), prior handling, elevated light levels, and small alterations in the apparatus structure have all been invoked as contributing factors to the variability in results (for a review, see Hogg, 1996). A brief review of the literature also shows wide variation in the age at which animals are tested, which suggests that ontogeny should be included with such factors as alterations in test conditions, strain differences, and so forth when interpreting the results from these studies.

\section{Gender Effects on Patterns of Escape}

There were no significant sex-related differences in the rates at which males and females escaped the apparatus or in the rate at which they either jumped or backed off the apparatus. Furthermore, no sex-related differences were found in three of the escape-related behaviors - that is, backtracking, attempting to back off, and preparing to jump. This finding supports those previous studies that showed no sex differences in active defense behaviors to a present threat source (D. C. Blanchard \& R. J. Blanchard, 1990; R. J. Blanchard et al., 1986). Clear dissimilarities were, however, found between the sexes in a number of other behaviors that have also been linked with escape (King, 1999a): Females scanned over the sides, reached the ends of the arms, and turned more than did males. A possible explanation for this apparent discrepancy may be found in the fact that at least two of these behaviors have also been shown to relate to motor/ exploratory/assessment activities (King, 1999a): A number of studies have reported a generally higher level of motor/exploratory activity in females than in males over a range of behavioral tests (Archer, 1975; Slob, Huizer, \& Van der Werff, 1986). Hence, an overall elevation of activity would be expected to yield higher rates of all behaviors, apart, it seems, from those most directly related to the escape itself - that is, backtracking, attempting to back off, and preparing to jump. In addition, Russell (1977) has reported that female rats respond to a short (10-min) exposure to novelty with higher levels of exploratory/information-gathering activities independent of motor activity than do males; scanning and turning are both information-gathering activities that do not involve translational movement.

It is interesting to note that the Blanchards and their coworkers have reported that gender differences in defensiveness in another potentially dangerous environmentthat is, the anxiety/test battery-also focus specifically on risk assessment behaviors, such as scanning, with females exhibiting consistently higher scores on these measures than males (e.g., D. C. Blanchard et al., 1991). As evidence is accruing that these more ethological measures may provide a more sensitive response to anxiolytic drugs than do conventional measures in other behavioral tests of anxiety-for example, the stable elevated plus maze (Cole et al., 1995; Cole \& Rodgers, 1994) - the finding of selective gender differences in defensive behaviors in this study adds support to the suggestion that females should be included more widely in preclinical psychopharmacological studies of anxiety (D. C. Blanchard, Griebel, \& R. J. Blanchard, 1995).

\section{Differential Behavioral Profiles \\ of Two Modes of Escape}

The animals in this experiment exhibited two clearly dissociable means of fleeing the aversive situation: Although a small proportion of the animals that escaped jumped off the apparatus, the majority used an alternative mode of escape - that is, backing off. In line with previous studies (King, 1999a, 1999b), the animals that jumped also prepared to jump, scanned, and end-reached significantly more frequently than did those that did not escape. In common with the animals that jumped, the animals that backed off also showed an increased propensity to scan over the sides of the arms. However, they did not reach 
the ends of the arms or prepare to jump any more frequently than did those animals that did not escape. They did, however, show an increased propensity to make $90^{\circ}$ turns and to backtrack along the arms, and they frequently made attempts to back off the apparatus before actually going over the side. It appears, therefore, that rats have access to an alternative repertoire of escape-related behaviors that shares only one common feature with jumping off and that possesses a number of distinct characteristics.

The finding of two discrete modes of escape in this study appears to be discrepant with previous studies in which all the animals that escaped did so by jumping off the apparatus (King, 1999a, 1999b). One explanation often invoked for the disparity in results seen in different studies hinges on such factors as alterations in experimental protocol or in the apparatus used. Although the experimental conditions (e.g., lighting levels, time of testing, previous handling, etc.) pertaining to the earlier studies were maintained in the present experiment, one aspect of the apparatus was changed. The screen surrounding the apparatus in the early studies was made from matt black wood and slightly angled away from the ends of the arms, thus presenting a solid, steeply inclined surface, although one well beyond reaching distance of the subjects. For ease of use, this was replaced in the present experiment by a vertical black cloth screen. It is unlikely, however, that this change would have resulted in any alterations in the animals' choice of escape mode, since a large proportion of the animals that jumped in the previous experiments did so from the center or the innermost portion of the arms.

A reexamination of the videotapes from these early studies does, however, suggest a more likely explanation for the apparent discrepancy. Because a novel behavioral test was being evaluated, very stringent criteria were adopted in these studies for any activity to be accepted as a mode of escape. Consequently, any behaviors that resulted in the animal's leaving the apparatus other than by the unequivocal act of jumping off were, according to these criteria, classified as falling off. Hence, a number of animals that would, in the present study, have been classified as backing off were placed back on the apparatus and then either stayed on for the remainder of the trial (to be included in the no-escape group), or subsequently jumped off at a later stage (to be included in the escape group). The recognition and classification, in this study, of an alternative repertoire of escape-related behaviors that appears to be relatively unaffected by either maturational or hormonal factors must be cause for optimism in the search for robust measures of fear/anxiety-related behaviors.

\section{REFERENCES}

ARCHER, J. (1975). Rodent sex differences in emotional and related behavior. Behavioral Biology, 14, 451-479.

Bernstein, S., \& Mason, W. A. (1962). The effects of age and stimulus conditions on the emotional responses of rhesus monkeys: Responses to complex stimuli. Journal of Genetic Psychology, 101, 279-298.

Blanchard, D. C., \& BlanChaRD, R. J. (1990). Effects of ethanol, benzodiazepines and serotonin compounds on ethopharmacological models of anxiety. In N. McNaughton \& G. Andrews (Eds.), Anxiety (pp. 188-199). Dunedin, New Zealand: University of Otago Press. Blanchard, D. C., BlanchaRd, R. J., Tom, P., \& RodGers, R. J. (1990). Diazepam changes risk assessment in an anxiety/defense test battery. Psychopharmacology, 101, 511-518.

Blanchard, D. C., Griebel, G., \& Blanchard, R. J. (1995). Gender bias in the preclinical psychopharmacology of anxiety: Male models for (predominantly) female disorders. Journal of Psychopharmacology, 9, 79-82.

Blanchard, D. C., Shepherd, J. K., Carobrez, A. P., \& Blanchard, R. J. (1991). Sex effects in defensive behavior: Baseline differences and drug interactions. Neuroscience \& Biobehavioral Reviews, 15, 461-468.

BlanChard, R. J., \& Blanchard, D. C. (1989). Anti-predator defensive behaviors in a visible burrow system. Journal of Comparative Psychology, 103, 70-82.

Blanchard, R. J., BlanChard, D. C., \& Weiss, S. M. (1990). Ethanol effects in an anxiety/defense test battery. Alcohol, 7, 375-381.

Blanchard, R. J., Flannelly, K. J., \& Blanchard, D. C. (1986). Defensive behaviors of laboratory and wild Rattus norvegicus. Journal of Comparative Psychology, 100, 101-107.

Blanchard, R. J., YudKo, E. B., Rodgers, R. J., \& BlanchaRd, D. C. (1993). Defense system psychopharmacology: An ethological approach to the pharmacology of fear and anxiety. Behavioural Brain Research, 58, 155-165.

Bolles, R. C., \& Woods, P. J. (1964). The ontogeny of behaviour in the albino rat. Animal Behaviour, 12, 427-441.

Bronstein, P. M., \& Hirsch, S. M. (1976). Ontogeny of defensive reactions in Norway rats. Journal of Comparative \& Physiological Psychology, 90, 620-629.

Cole, J. C., Burroughs, G. J., Laverty, C. R., Sheriff, N. C., Sparham, E. A., \& Rodgers, R. J. (1995). Anxiolytic-like effects of yohimbine in the murine plus maze: Strain independence and evidence against alpha 2-adrenoceptor mediation. Psychopharmacology, $118,425-436$

Cole, J. C., \& Rodgers, R. J. (1994). Ethological evaluation of the effects of acute and chronic buspirone treatment in the murine elevated plus-maze test: Comparison with haloperidol. Psychopharmacology, 114, 288-296.

Crepeau, L. J., \& Newman, J. D. (1991). Gender differences in reactivity of adult squirrel monkeys to short-term environmental challenges. Neuroscience \& Biobehavioral Reviews, 15, 469-471

GREEN, P. C. (1965). Influence of early experience and age on expression of affect in monkeys. Journal of Genetic Psychology, 106, 157-171.

HANDLEY, S. L., \& MITHANI, S. (1984). Effects of alpha-adrenoceptor agonists and antagonists in a maze-exploration model of 'fear'-motivated behavior. Naunyn-Schmiedeberg's Archives of Pharmacology, 327, $1-5$.

HARD, E., EnGEL, J., \& MuSi, B. (1982). The ontogeny of defensive reactions in the rat: Influence of the monoamine transmission systems. Scandinavian Journal of Psychology, Suppl. 1, 90-96.

HoGG, S. (1996). A review of the validity and variability of the levated plus-maze as an animal model of anxiety. Pharmacology, Biochemistry \& Behavior, 54, 21-30.

KING, S. M. (1999a). Escape-related behaviours in an unstable elevated and exposed environment: I. A new behavioral model of extreme anxiety. Behavioural Brain Research, 98, 113-126.

KING, S. M. (1999b). Escape-related behaviours in an unstable elevated and exposed environment: II. Long-term sensitization after repetitive electrical stimulation of the rodent midbrain defense system. Behavioural Brain Research, 98, 127-142.

KING, S. M., GreEN, S., \& VALE, A. (1999). Anxiogenic and anxiolytic drug effects on escape-related behaviours in the unstable elevated plus maze: Concomitant effects on locomotor activity. Society for Neuroscience Abstracts, 25, 2135.

KuhlmanN, F. (1909). Some preliminary observations on the development of instincts and habits in young birds. Psychological Monographs, 11 (1), 49-84.

MELZACK, R. (1954). The genesis of emotional behavior: An experimental study of the dog. Journal of Comparative \& Physiological Psychology, 47, 166-168.

Murphey, R. M., BeCaK, W., \& Dias de Moraes, A. E. (1981). Escape 
efficiency of diploid and polyploid frogs: A comparison of Odontophrynus cultripes and $O$. americanus. Behavior Genetics, 11, 255-265.

Rex, A., Sondern, U., Voigt, J. P., Franck, S., \& Fink, H. (1996). Strain differences in fear-motivated behavior of rats. Pharmacology, Biochemistry \& Behavior, 54, 107-111.

RuSSELl, P. A. (1977). Sex differences in rats' stationary exploration as a function of stimulus and environmental novelty. Animal Learning \& Behavior, 5, 297-302.

SCHALleR, G. B., \& EMLEN, J. T. (1962). The ontogeny of avoidance behavior in some precocial birds. Animal Behaviour, 10, 370-381.
Scouten, C. W., Grotelueschen, L. K., \& Beatty, W. W. (1975). Androgens and the organization of sex differences in active avoidance behavior in the rat. Journal of Comparative \& Physiological Psychology, 88, 264-270.

Slob, A. K., Huizer, T., \& VAN der WerfF, J. J. (1986). Ontogeny of sex differences in open field ambulation in the rat. Physiology \& Behavior, 37, 313-315.

(Manuscript received March 31, 1999; revision accepted for publication July 2, 1999.) 